\title{
EXTREMAL STRUCTURE IN OPERATOR SPACES
}

BY

\author{
M. SHARIR (1)
}

ABSTRACT. (a) A characterization of extreme operators (in the unit ball of operators) between $L^{1}$-spaces is given, together with other related properties. (b) A general theorem of Kre in-Milman type for the unit ball of operator spaces is proved, and is applied to operators between $L^{1}$-spaces and to operators into $C$-spaces.

1. Introduction. In this paper we study in detail extreme points in the unit ball of operator spaces (which we, shall briefly call extreme operators). Although we treat mostly operators in $L^{1}$-spaces and $C$-spaces, some of the results are general $(\$ 3)$. Before discussing the results, we go briefly over the notations. They are mostly standard and we refer to [14] for some of the notations, though we remark that if $E$ is a Banach space, the action of $x^{*} \in E^{*}$ on $x \in E$ will be denoted either by $x^{*}(x)$ or by $\left\langle x^{*}, x\right\rangle$. The canonical imbedding of $E$ into $E^{* *}$ will be denoted by $J_{E}$. The scalars are either real or complex; only in Theorem 2.10 are they assumed to be real. Generalizing a notation of Morris and Phelps [12], we call an operator $T: E \rightarrow F$, for two Banach spaces $E$ and $F$, a nice operator, if $T^{*}\left(\operatorname{ext} S\left(F^{*}\right)\right) \subset$ ext $S\left(E^{*}\right)$. It is easy to verify that every nice operator is extreme, and to construct examples where there exist no nice operators (e.g. in $\mathcal{L}\left(l^{1}, l^{2}\right)$ ). We refer to [1] for nontrivial examples of extreme nonnice operators into $C$-spaces (i.e. spaces of continuous functions over a compact Hausdorff space). By an $L^{1}$-space we denote a Banach space which is isometric to a space $L^{1}(S, \Sigma, \mu)$, in the notations of [4]. Since we shall deal only with Banach-space properties of such a space, we may assume, for example, that the dual of $L^{1}(S, \Sigma, \mu)$ is $L^{\infty}(S, \Sigma, \mu)$, or we may treat a $\sigma$-finite measure as a finite measure, since those properties can be obtained by an isometry. We shall usually write briefly $L^{1}(\mu)\left(L^{\infty}(\mu)\right)$ instead of $L^{1}(S, \Sigma, \mu)\left(L^{\infty}(S, \Sigma, \mu)\right)$. The dual of an $L^{1}$-space is a space $C(X)$, where $X$ is byperstonian, and the $L^{1}$-space may be

Received by the editors December 20, 1972.

AMS (MOS) subject classifications (1970). Primary 47D20, 47D15, 46E30; Secondary $46 \mathrm{E} 15$.

Key words and phrases. Extreme operators, nice operators, theorems of Krein-Milman type, $L^{1}$-spaces, hyperstonian spaces, $C$-spaces.

(1) This paper is a part of the author's Ph. D. thesis, which is being prepared at Tel-Aviv University, under the supervision of Dr. A. J. Lazar, whom the author wishes to thank for his faithful guidance and great help in the research. 
regarded as the space of normal measures on $X$. We take as known the basic properties of hyperstonian spaces and normal measures. They are to be found in [2], [7], or [16].

The problem of whether every extreme operator between $C$-spaces must be nice was presented in [1], and is still open. A dual problem, which arises natural$1 y$, is whether every extreme operator between $L^{1}$-spaces is nice. In $\$ 2$ we answer this problem in the affirmative (Theorem 2.2), and derive several other properties of extreme operators between $L^{1}$-spaces, between $C$-spaces, and with an $L^{1}$-space as their domain. In $\$ 3$ we prove (Theorem 3.1 ) that under very general assumptions on the Banach spaces $E$ and $F, \mathfrak{L}(E, F)$ is dense in $\mathscr{L}\left(F^{*}, E^{*}\right)$ with suitable natural topologies (the weak*-operator topology on $\mathscr{L}\left(F^{*}, E^{*}\right)$, which induces the weak-operator topology on $\mathcal{L}(E, F)$ ), where $\mathcal{L}(E, F)$ is injected into $\mathfrak{L}\left(F^{*}, E^{*}\right)$ by assigning to each operator its adjoint. As a conclusion we obtain a general theorem of Krein-Milman and Milman tyjpe (Theorem 3.2), giving a necessary and sufficient condition for $S(£(E, F))$ to be the weak-operator-closed convex hull of a subset $A$, in terms of the "nearness" of ext $S\left(\mathscr{L}\left(F^{*}, E^{*}\right)\right)$ to $A$. In $\$ 4$ we apply this theorem to operators between $L^{1}$-spaces, and find an equivalent simple condition for $S(\mathcal{L}(E, F))$ to be the weak-operator-closed convex hull of its extreme points, in terms of the extremal structure of $E, F$, and their decompositions into a sum of bomogeneous $L^{1}$-subspaces (Theorems $4.4,4.5,4.10$ ). We also obtain several results concerning open mappings of hyperstonian spaces. In $\S 4$ we give some other applications of the results of $\$ 3$. First we apply them to operators from an $l^{1}(\Gamma)$-space. Then we apply them to operators into $C$-spaces, and improve some results of Morris and Phelps [12].

2. Characterization of extreme operators in $L^{1}$-spaces. In this section we characterize the extreme operators between $L^{1}$-spaces as the nice operators. This is the dual problem of the still unsolved problem of a similar characterization of extreme operators between $C$-spaces (cf. [1]). The technique used here is a modification of a technique used in [14] to investigate the dual problem.

Lemma 2.1. Let $E$ be an $L^{1}$-space, $F$ an arbitrary Banach space and $T$ : $E \rightarrow F$ an extreme operator. Identify $E^{*}$ with $C\left(Y_{0}\right)$, for an appropriate byperstonian space $Y_{0}$. Then $\left\{y \in Y_{0} ;\left\|T^{* *} y\right\|=1\right\}$ is dense in $Y_{0}$.

Proof. (Cf. Theorem 1 of [14]). For each integer $n$, the set $G_{n}=\left\{y \in Y_{0}\right.$; $\left.\left\|T^{* *} y\right\|>1-1 / n\right\}$ is open in $Y_{0}$. To see that it is dense there, take any $g \epsilon$ $C\left(Y_{0}\right), 0 \leq g \leq 1$, such that $\left.g\right|_{G_{n}} \equiv 0$. Let $p \in S(F)$ be a nonzero element and define an operator $R: E \rightarrow F$ by

$$
R \mu=n^{-1}\langle g, \mu\rangle p, \quad \mu \in E .
$$


Then

$$
R^{* *} y=n^{-1} g(y) \hat{\phi}, \quad y \in Y_{0},
$$

where $\hat{p}$ is the canonical image of $p$, and $\|T \pm R\|=\sup _{y \in Y_{0}}\left\|T^{* *} y \pm R * *_{y}\right\| \leq 1$. Thus $R=0$ and $g=0$ and $G_{n}$ is therefore dense in $Y_{0}$. Hence,

$$
\left\{y \in Y_{0} ;\left\|T^{* *} y\right\|=1\right\}=\bigcap_{n \geq 1} G_{n}
$$

is also dense. Q.E.D.

Theorem 2.2. Let $E, F$ be $L^{1}$-spaces; then every extreme operator $T: E \rightarrow$ $F$ is nice.

Proof. Let $E^{*}=C\left(Y_{0}\right), F^{*}=C\left(X_{0}\right)$, and put $H=\left\{y \in Y_{0} ;\left\|T^{* *} y\right\|=1\right\}$. Since $H$ is dense in $Y_{0}$, and $Y_{0}$ is extremally-disconnected compact, we have $Y_{0}=\beta H$ (the Stone-Čech compactification of $H$ ) (cf. [5, p. 96]). Let $A \subset X_{0}$ be clopen (i.e. closed and open). Define the following $w^{*}$-continuous mappings $R_{1}^{*}, R_{2}^{*}: Y_{0}$ $\rightarrow C\left(x_{0}\right) *$ by

$$
R_{1}^{*} y=\left.T^{* *} y\right|_{A}, \quad R_{2}^{*} y=\left.T^{* *} y\right|_{A^{\prime}}, \quad y \in Y_{0} .
$$

They induce operators $R_{1}, R_{2}: C\left(X_{0}\right) \rightarrow C\left(Y_{0}\right)$ which are $w^{*}$-continuous, since, e.g., $R_{1} f=T^{*}\left(\chi_{A} f\right), f \in C\left(X_{0}\right)$; and multiplication is $w^{*}$-continuous on $C\left(X_{0}\right)$. Now consider the functions $y \rightarrow\left\|R_{i}^{*} y\right\|, y \in Y_{0}, i=1,2$. These are lower semicontinuous functions on $Y_{0}$. But if $y \in H$, then $\left\|R_{1}^{*} y\right\|+\left\|R_{2}^{*} y\right\|=\left\|T^{* *} y\right\|=1$. Hence these functions are continuous on $H$. Let $\phi_{i}$ be the unique continuous extension of $\left\|R_{i}^{*}(\cdot)\right\|$ to $Y_{0}, i=1,2$. Define a w*-continuous mapping $R^{*}: Y_{0} \rightarrow C\left(X_{0}\right) *$ by

$$
R^{*} y=\phi_{1}(y) R_{2}^{*} y-\phi_{2}(y) R_{1}^{*} y, \quad y \in Y_{0} .
$$

The operator $R: C\left(X_{0}\right) \rightarrow C\left(Y_{0}\right)$ induced by $R^{*}$ is simply $R f=\phi_{1} R_{2} f-\phi_{2} R_{1} f$. $f \in C\left(X_{0}\right)$, and is therefore a $w^{*}$-continuous operator. Hence $R=K^{*}$, for some $K \in \mathfrak{L}(E, F)$ and we have

$$
\|T \pm K\|=\sup _{y \in Y_{0}}\left\|T_{y}^{* *} \pm R^{*} y\right\| .
$$

A standard computation yields for $y \in H$ that $\left\|T^{* *} y \pm R^{*} y\right\| \leq 1$, and by the $w^{*}$ compactness of $S\left(C\left(X_{0}\right) *\right)$, the $w^{*}$-continuity of $T^{* *} \pm R^{*}$, and the fact that $H$ is dense in $Y_{0}$, we obtain $\|T \pm K\| \leq 1$. Hence $K=0$, which means, for $y \in H$,

$$
0=\left\|R_{y}^{*}\right\|=2\left\|\left.T_{y}^{* *}\right|_{A}\right\| \cdot\left\|\left.T_{y}^{* *}\right|_{A^{\prime}}\right\| \cdot
$$

That is, for every $y \in H, T^{* *} y$ is either supported in $A$, or in $A^{\prime}$. Again, by continuity, this is true for every $y \in Y_{0}$. But since $A$ was an arbitrary clopen subset 
of $X_{0}$, it follows that, for each $y \in Y_{0}, T^{* *} y$ has a one-point support. Hence, for each $y \in H$,

$$
T^{* *} y \in\left\{\lambda \delta_{x} ;|\lambda|=1, x \in X_{0}\right\} \equiv \operatorname{ext} S\left(C\left(x_{0}\right)^{*}\right),
$$

and by the $\mathrm{w}^{*}$-compactness of this set, we have $T^{* *}\left(Y_{0}\right) \subset$ ext $S\left(C\left(X_{0}\right) *\right)$. Thus we have shown that $T^{*}$ is a nice operator. To see that $T$ itself is nice, take any $f \in$ ext $S\left(C\left(X_{0}\right)\right)$ (namely, $|f| \equiv 1$ ). Then $\left|T^{*} f(y)\right|=\left|T^{* *} y(f)\right|=1$, for every $y \in Y_{0}$, and therefore $T^{*} f \in \operatorname{ext} S\left(C\left(Y_{0}\right)\right)$, and $T$ is nice. Q.E.D.

Corollary 2.3. The composition of two extreme operators between $L^{1}$-spaces is always extreme. In particular, if $E$ is an $L^{1}$-space, then the set of extreme operators in $\mathcal{L}(E, E)$ forms a semigroup with identity, under composition.

Proof. Trivial, since a composition of nice operators is always nice. Also note, that the identity operator on any Banach space is always extreme, since it is nice. Q.E.D.

Corollary 2.4. Let $E, F$ be $L^{1}$-spaces. Then the following statements are equivalent for an operator $T: E \rightarrow F$.

(a) $\left(T^{*}\right)^{n}$ is extreme for some $n \geq 0$.

(b) $\left(T^{*}\right)^{n}$ is nice for some $n \geq 0$.

(c) $\left(T^{*}\right)^{n}$ is extreme for every $n \geq 0$.

(d) $\left(T^{*}\right)^{n}$ is nice for every $n \geq 0$.

(By $\left(T^{*}\right)^{n}$ we mean the nth order adjoint operator of $T$. )

Proof. The implications (d) $\Rightarrow(b) \Rightarrow$ (a) and (d) $\Rightarrow(c) \Rightarrow$ (a) hold for any pair of Banach spaces. What remains to show is that $(a) \Rightarrow(d)$. Now it is true, in general, that if $T^{*}$ is extreme, then $T$ is also extreme. Hence, (a) implies that $T$ is extreme. By the proof of Theorem 2.2 we know that $T$ is nice and also that $T^{*}$ is nice. Thus the proof will be completed if we show that, if $R$ is a nice operator between $C$-spaces, then $R^{*}$ is extreme, thus obtaining (d) inductively, for the second dual of an $L^{1}$-space is again an $L^{1}$-space. We have even the following stronger result, which generalizes Corollary 2 in [14]:

Lemma 2.5. Let $E$ be a Banach space, $Y$ a compact Hausdorff space, and $T$ an operator in $\mathcal{L}(E, C(Y))$. Then $T$ is nice if and only if $T^{*}$ is extreme.

Proof. Put $C\left(Y_{0}\right)=C(Y)^{* *}, J=J_{C(Y)}$. There is a natural isometry between $\mathcal{L}\left(E, C\left(Y_{0}\right)\right)$ and $\mathcal{L}\left(C(Y)^{*}, E^{*}\right)$, in which $J T$ corresponds to $T^{*}$. Now, if $T$ is nice, then $J T$ is also nice (since $J$ is a nice operator), and therefore $T^{*}$ is extreme. On the other hand, if $T^{*}$ is extreme, then $J T: E \rightarrow C\left(Y_{0}\right)$ is also extreme. Thus $(J T)^{*} y$ is extreme in $S\left(E^{*}\right)$ for every isolated $y \in Y_{0}$ (cf. [1]). But if $\hat{y} \in Y_{0}$ is isolated, then $\delta_{\hat{y}}$ is a normal measure (cf. [2]), extreme in $S\left(C\left(Y_{0}\right) *\right)$, 
hence it is extreme in $S\left(C(Y)^{*}\right)$, and is therefore of the form $\delta_{y}$, for some $y \in Y$. Conversely, for each $y \in Y, \delta_{y}$, viewed as an extreme normal measure on $Y_{0}$, is easily seen to have a one-point support, and is therefore of the form $\delta_{\hat{y}}$, for some isolated $\hat{y} \in Y_{0}$, and we have $J^{*} \hat{y}=y$. Hence, $T^{*} y=(J T)^{*} \hat{y} \in \operatorname{ext} S\left(E^{*}\right)$, for each $y \in Y$. Hence $T$ is nice. Q.E.D.

Corollary 2.6. Let $X, Y$ be compact Hausdorff spaces, and $T$ an operator in $\mathcal{L}(C(X), C(Y))$. Then the following statements are equivalent.

(a) $\left(T^{*}\right)^{n}$ is extreme for some $n \geq 1$.

(b) $\left(T^{*}\right)^{n}$ is nice for some $n \geq 0$.

(c) $\left(T^{*}\right)^{n}$ is extreme for every $n \geq 0$.

(d) $\left(T^{*}\right)^{n}$ is nice for every $n \geq 0$.

Proof. Again we have immediately (d) $\Rightarrow(\mathrm{c}) \Rightarrow$ (a) and (d) $\Rightarrow$ (b). (b) $\Rightarrow$ (a) is obvious if $n \geq 1$, and follows from Lemma 2.5 if $n=0$.

(a) $\Rightarrow$ (d). It follows from (a) that $T^{*}$ is extreme. Since $T^{*}$ is an operator between $L^{1}$-spaces, we have from Corollary 2.4 that $\left(T^{*}\right)^{n}$ is nice for every $n \geq 1$. For $n=0$ this follows from Lemma 2.5. Q.E.D.

Remark. It would be nicer if we could put $n \geq 0$ in statement (a). However, this could be done only if we knew that every extreme operator between $C$-spaces is nice, which is still an open problem.

An application of Theorem 2.2 to extreme invariant operators between $L^{1}$. spaces has been pointed out by S. Reich:

Remark (S. Reich). Let $E$ be a Banach space and $\Sigma$ a topological semigroup. We will say that $\Sigma$ acts on $E$ from the left if there is a separately continuous map $\Sigma \times E \rightarrow E$, denoted by $(s, x) \rightarrow s x$, such that the operators $\{x \rightarrow s x$ : $s \in \Sigma\}$ belong to the unit ball of $\mathcal{L}(E, E)$, and $(s t) x=s(t x)$ for all $s, t$ in $\Sigma$ and $x$ in $E$. If $F$ is another Banach space, then an operator $T: E \rightarrow F$ is said to be invariant if $T(s x)=T x$ for all $s \in \Sigma$ and $x \in E$. Put $B\left(E^{*}\right)_{\Sigma}=\left\{y \in E^{*}: y\right.$ is invariant and $\|y\| \leq 1\}$, and more generally, $B(E, F)_{\Sigma}=\{T \in \mathscr{L}(E, F): T$ is invariant and $\|T\| \leq 1\}$. In this setting, Theorem 2.2 implies the following result: Let $E$ and $F$ be $L^{1}$-spaces and $\Sigma$ a topological semigroup which acts on $E$ from the left. Suppose that RUC $(\Sigma)$, the space of right uniformly continuous bounded scalar-valued functions on $\Sigma$, has a right invariant mean. Then $T \in B(E, F)_{\Sigma}$ is extreme there if and only if $T^{*} f$ is extreme in $B\left(E^{*}\right)_{\Sigma}$ for each extreme $f$ in $S\left(F^{*}\right)$.

Proof. We may assume that $E_{\Sigma}^{*}=\left\{y \in E^{*}: y\right.$ is invariant $\}$ is not trivial. If $x$ is in $E$ and $f$ in $E^{*}$, then the function $b=b(x, f)$ defined on $\Sigma$ by $b(s)=\langle f, s x\rangle$ is in RUC $(\Sigma)$. Therefore we can define, for each $f \in E^{*}$, a functional $P f$ on $E$ by $\langle P f, x\rangle=m(b(x, f))$, where $m$ is a (fixed) right invariant mean on $\operatorname{RUC}(\Sigma)$. Clearly $P f \in E^{*}$ and $\|P f\| \leq\|f\|$. If $f \in E_{\Sigma}^{*}$, then $\langle P f, x\rangle=m(s \rightarrow\langle f, s x\rangle)=$ 
$m(s \rightarrow\langle f, x\rangle)=\langle f, x\rangle$, for all $x \in E$. Finally, if $t \in \Sigma$, then $\langle P f, t x\rangle=m(b(t x, f))=$ $m(s \rightarrow\langle f, s t x\rangle)=m(s \rightarrow\langle f, s x\rangle)=\langle P f, x\rangle$, because $m$ is right-invariant. Thus $P$ is a projection of norm 1 from $E^{*}$ onto $E_{\Sigma}^{*}$. Therefore its range can be identified with $C(K)$ for some stonian $K$. But since $E_{\Sigma}^{*}$ is $\mathrm{w}^{*}$-closed, $K$ is hyperstonian and there is an $L^{1}$-space $G$ such that $G^{*}=E_{\Sigma}^{*}$, with the same $w^{*}$-topology on their unit balls. Hence, $B(E, F)_{\Sigma}$ can be identified with $S(\mathcal{\varrho}(G, F))$ and the result follows.

We give now another application of Lemma 2.1.

Theorem 2.7. Let $E$ be an $L^{1}$-space, $F$ a Banach space sucb that $F^{* *}$ is strictly convex, and $T$ an operator in $\mathcal{L}(E, F)$. Then the first three following statements are equivalent and imply the fourth one.

(a) $T$ is extreme.

(b) $T^{*}$ is extreme.

(c) There is a dense subset $H$ of $Y_{0}$ (where $C\left(Y_{0}\right)=E^{*}$ ) such that $T^{* *}(H)$ $C \operatorname{ext} S\left(F^{* *}\right)$.

(d) $T(\operatorname{ext} S(E)) \subset \operatorname{ext} S(F)$.

Proof. (a) $\Rightarrow(\mathrm{c})$. Since $F^{* *}$ is strictly-convex, this follows immediately from Lemma 2.1 .

(c) $\Rightarrow$ (b). Immediate (cf. a remark in [1]).

(b) $\Rightarrow$ (a). True in general.

(c) $\Rightarrow$ (d). Let $p \in \operatorname{ext} S(E)$. Then $\hat{p}=\delta_{y}$, for some isolated $y \in Y_{0}$. Obviously $y \in H$, and therefore $\widehat{T p}=T^{* *} y \in \operatorname{ext} S\left(F^{* *}\right)$. Hence $T p \in \operatorname{ext} S(F)$. Q.E.D.

The implication (a) $\Rightarrow$ (d) of the previous theorem can be proved in general, with the aid of the following simple lemma:

Lemma 2.8. Let $E$ be a Banacb space, and $\Gamma$ a set. An operator $T \epsilon$ $\mathfrak{L}\left(l^{1}(\Gamma), E\right)$ is extreme if and only if $T e_{\gamma} \in$ ext $S(E)$ for each $\gamma \in \Gamma$. (e, are the unit vectors in $l^{1}(\Gamma)$.)

Proof. If there is $\gamma_{0} \in \Gamma, 0 \neq p \in E$, such that $\left\|T e_{\gamma_{0}} \pm p\right\| \leq 1$, then define $U \in \mathfrak{L}\left(l^{1}(\Gamma), E\right)$ by $U x=x\left(\gamma_{0}\right) p, x \in l^{1}(\Gamma)$. Then $\|T \pm U\| \leq 1$, and $T$ is not extreme. Conversely, if $T e_{\gamma} \in \operatorname{ext} S(E)$ for each $\gamma \in \Gamma$, then $\|T \pm U\| \leq 1$ implies that $U e_{y}=0$ for each $\gamma \in \Gamma$, and therefore $U=0$. Q.E.D.

Theorem 2.9. Let $E$ be an $L^{1}$-space, $F$ a Banach space, and $T$ an extreme operator in $\mathcal{L}(E, F)$. Then $T($ ext $S(E)) \subset$ ext $S(F)$.

Proof. Looking at $E$ as the space of normal measures on $Y_{0}$, we can write $E=\left(E_{1} \oplus E_{2}\right)_{1}$, where $E_{1}$ is the subspace of purely nonatomic measures and $E_{2}$ is the subspace of purely atomic measures. By the remark at the proof of

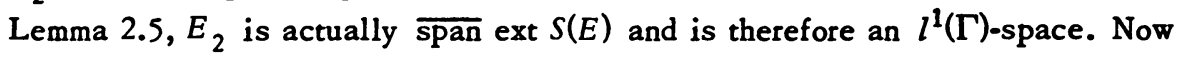


$\left.T\right|_{E_{2}}$ is extreme in $\mathscr{\sim}\left(E_{2}, F\right)$, for if there is $U \in \mathscr{L}\left(E_{2}, F\right)$ such that $\left\|\left.T\right|_{E_{2}} \pm U\right\|$ $\leq 1$, then define $\tilde{U} \in \mathcal{L}(E, F)$ by

$$
\tilde{U}\left(x_{1}+x_{2}\right)=U x_{2}, \quad x_{i} \in E_{i}, i=1,2 .
$$

Then $\|T \pm \tilde{U}\| \leq 1$ and therefore $U=0$. By Lemma 2.8 we have $T(\operatorname{ext} S(E))=$ $\left.T\right|_{E_{2}}\left(\operatorname{ext} S\left(E_{2}\right)\right) C \operatorname{ext} S(F)$. Q.E.D.

We end this section with an interesting property of the space $L^{1}(\mu, E)$, where $E$ is a Banach space. This is the space of $E$-valued functions whose norm is $\mu$-integrable (cf. [4, p. 121]). We refer here to real Banach spaces $E$ satisfying the (equivalent) conditions of Theorem 4.7 of [10]. The condition we make use of is

(2.1) For each $p \in$ ext $S(E), m \in$ ext $S\left(E^{*}\right),|m(p)|=1$.

A weaker condition (which is the one actually mentioned in [10]) is

(2.2) There is $p \in$ ext $S(E)$ such that, for each $m \in \operatorname{ext} S\left(E^{*}\right),|m(p)|=1$.

It is equivalent to

(2.3) There is a compact Hausdorff space $K$ such that $E$ is isometrically imbedded as a separating subspace of $C(K)$ containing 1.

Theorem 2.10. Let $L^{1}(\mu)$ be an $L^{1}$ space with atoms, and $E$ a Banach space. Then $E$ satisfies (2.1) (or (2.2)) if and only if $L^{1}(\mu, E)$ satisfies the same condition.

Proof. We treat only condition (2.1). The other condition is treated similarly. The extreme points of $S\left(L^{1}(\mu, E)\right)$ were shown in [15] to be the points of the form $q= \pm \chi_{A} p / \mu(A)$, where $p \in \operatorname{ext} S(E)$ and $A$ is an atom. Now, $L^{1}(\mu, E) *$ can be identified isometrically with $\mathscr{L}\left(L^{1}(\mu), E^{*}\right)$ and with $\mathscr{\sim}\left(E, C\left(Y_{0}\right)\right)$, where $L^{1}(\mu) *=C\left(Y_{0}\right)$ (cf. [6]). Let $T \in \underset{\sim}{\operatorname{ext}} S\left(L^{1}(\mu, E) *\right)$ and $\tilde{T} \epsilon \operatorname{ext} S\left(\mathscr{Q}\left(E, C\left(Y_{0}\right)\right)\right)$ be the corresponding operator. Since $\tilde{T}$ is extreme, and $Y_{0}$ is extermally-disconnected, we have by [14] that $\tilde{T}^{*}\left(Y_{0}\right) \subset\left(\text { ext } S\left(E^{*}\right)\right)^{-w^{*}}$. Now, if $E$ has property (2.1), pick $T \in \operatorname{ext} S\left(L^{1}(\mu, E) *\right), q= \pm \chi_{A} p / \mu(A) \in \operatorname{ext} S\left(L^{1}(\mu, E)\right)$. Then

$$
|\langle T, q\rangle|=\left|\left\langle\tilde{T} p, \chi_{A} / \mu(A)\right\rangle\right| \text {. }
$$

But $|\tilde{T} p(y)|=\left|\tilde{T}^{*} y(p)\right|=1$, for each $y \in Y_{0}$, by condition (2.1). Hence $\tilde{T} p$, as an element in $L^{\infty}(\mu)$, is a constant of absolute value 1 on $A$ since $A$ is an atom, and therefore $|\langle T, q\rangle|=1$, and so $L^{1}(\mu, E)$ satisfies condition (2.1).

Conversely, if $L^{1}(\mu, E)$ satisfies (2.1), let $p \in \operatorname{ext} S(E), m \in$ ext $S\left(E^{*}\right)$ and $\phi \in \underset{\sim}{\operatorname{ext}} S\left(L^{1}(\mu)\right)$. Then $\phi p \in \operatorname{ext} S\left(L^{1}(\mu, E)\right)$. The operator $\widetilde{T}: E \rightarrow C\left(Y_{0}\right)$, defined by $\tilde{T} x=m(x) 1, x \in E$, is extreme, since it is nice, and is therefore corresponding to a point $T \in \operatorname{ext} S\left(L^{1}(\mu, E)^{*}\right)$. Therefore $1=|\langle T, \phi p\rangle|=|\langle\tilde{T} p, \phi\rangle|=|m(p)|$, and so $E$ satisfies (2.1). Q.E.D. 
3. A theorem of Kreĭn-Milman type for operator spaces. The recent results on the principle of local reflexivity (cf. [8]) yield a general form of a theorem of Krein-Milman and Milman type for the unit ball of operator spaces. Let $E, F$ be Banach spaces. Denote by $\phi: \mathfrak{L}(E, F) \rightarrow \mathcal{L}\left(F^{*}, E^{*}\right)$ the natural injection such that $\phi(T)=T^{*}$, for $T \in \mathscr{L}(E, F)$. Consider the weak-operator (abbreviated w.o.) topology on $\mathscr{L}(E, F)$ (cf. [4, p. 476]), and the weak*-operator (abbreviated w*.o.) topology on $\mathcal{L}\left(F^{*}, E^{*}\right)$, i.e., the topology in which $T_{a} \rightarrow T$ if and only if $T_{a} y^{*} \stackrel{\mathbb{W}^{*}}{\longrightarrow} T y^{*}$, for each $y^{*} \in F^{*}$. In those topologies it is clear that $\phi$ is a homeomorphism into $\mathscr{L}\left(F^{*}, E^{*}\right)$. Also trivially, $\phi(S(\mathscr{L}(E, F))) \subset S\left(\left(^{*}, F^{*}\right)\right)$. Recall also that a Banach space $E$ has the metric approximation property (M.A.P.) iff for each $\epsilon>0$ and each finite-dimensional $H \subset E$ there is a finite-rank operator $T: E \rightarrow E$, of norm $\leq 1+\epsilon$, such that $T x=x$, for each $x \in H$ (cf. [6]).

Theorem 3.1 (a density theorem). If either $E$ or $F^{*}$ has the M.A.P., then $\phi(S(\mathscr{Q}(E, F)))$ is $w^{*}$.o. -dense in $S\left(\complement^{*}\left(F^{*} \cdot E^{*}\right)\right)$.

Proof. (a) Assume that $E$ has the M.A.P. Since we are dealing with locally convex topologies, it is sufficient to prove that for each $\epsilon>0$ and each w*.o.continuous linear functional $\mu$ on $\mathcal{L}\left(F^{*}, E^{*}\right)$ with $\|\mu\|=1$, there is $T \in S(\mathscr{Q}(E, F))$ such that $\left|\mu\left(T^{*}\right)\right|>1-\epsilon$. If $\mu$ is such a functional, then there are $y_{1}^{*}, \ldots, y_{n}^{*} \in F^{*}$ : $x_{1}, \ldots, x_{n} \in E$, such that $\mu(R)=\sum_{i=1}^{n}\left\langle R y_{i}^{*}, x_{i}\right\rangle, R \in \mathscr{L}\left(F^{*}, E^{*}\right)$. Since $\|\mu\|=1$ there is $R \in S\left(Q^{*}\left(F^{*}, E^{*}\right)\right)$, such that $\mu(R)>1-\epsilon / 2$. Since $E$ has the M.A.P., there is a finite-dimensional subspace $G \subset E$ and an operator $Q: E \rightarrow G$, such that $Q x_{i}=x_{i}$, for each $1 \leq i \leq n$, and $\|Q\| \leq 1+\epsilon / 4$. Regarding $Q$ as an operator in $E, Q^{*}$ is also a finite-rank operator, and so is $Q^{*} R: F^{*} \rightarrow E^{*}$. Denote its range by $H$, and let $L$ be the subspace of $F^{*}$ spanned by $y_{1}^{*}, \ldots, y_{n}^{*}$. Then, by [8, Lemma 3.1], there is a weak*-continuous operator $U: F^{*} \rightarrow H$, such that $\|U\| \leq 1+\epsilon / 2$, and $\left.U\right|_{L}=\left.Q^{*} R\right|_{L}$. Now, $U$ is also weak*-continuous, as an operator from $F^{*}$ into $E^{*}$, and so $U=V^{*}$, for some $V \in \mathscr{L}(E, F)$. But it is easy to see that $\mu\left(V^{*}\right)=\mu\left(Q^{*} R\right)=\mu(R)$, and so $T=V /(1+\epsilon / 2)$ is the required operator.

(b) If $F^{*}$ has the M.A.P., let $\mu, R, \epsilon$ be as above. Then there is a finitedimensional subspace $G \subset F^{*}$, and an operator $Q: F^{*} \rightarrow G$, with $\|Q\| \leq 1+\epsilon / 4$, such that $Q y_{i}^{*}=y_{i}^{*}$, for each $1 \leq i \leq n$. Again, by [8] there is an operator $Q_{0}$ : $F \rightarrow F$, such that $\left\|Q_{0}\right\| \leq 1+\epsilon / 2, Q_{0}^{*}$ has its range in $G$, and $Q_{0}^{*} y_{i}^{*}=y_{i}^{*}$ for each $1 \leq i \leq n$. Hence $\mu\left(R Q_{0}^{*}\right)=\mu(R)$. But $R Q_{0}^{*}$ is weak*-continuous, since $Q_{0}^{*}$ sends $w^{*}$ converging nets to norm-converging nets, and therefore $R Q_{0}^{*}=U^{*}$, for some $U \in \mathscr{L}(E, F)$, and $T=U /(1+\epsilon / 2)$ is the required operator. Q.E.D.

Remarks. (1) Under no assumptions on $E$ or $F, \phi(£(E, F))$ is always $w^{*}$.o.-dense in $\mathcal{L}\left(F^{*}, E^{*}\right)$. Indeed, in this case we have to show that if $\mu$ is a $w^{*}$.o.-continuous functional vanishing on $\phi(£(E, F))$, then $\mu$ is identically zero, 
and this is done directly, using the fact that in a Banach space there is always a projection on every finite-dimensional subspace. (2) The classical Goldstine theorem [4, p. 424] is a special case of Theorem 3.1, by taking $E$ to be one-dimensional.

As a corollary of Theorem 3.1 we have our central result:

Theorem 3.2 (a theorem of Krein-Milman type). Let E, $F$ be Banach spaces such that either $E$ or $F^{*}$ bas the M.A.P. Let $S_{0}=S(£(E, F)), S_{1}=S\left(£\left(F^{*}, E^{*}\right)\right)$, and $A$ a subset of $S_{0}$. Then $S_{0}=\overline{\mathrm{conv}}^{\mathrm{w} \cdot 0 \cdot A}$ if and only if ext $S_{1} \subset(\phi(A))^{-\mathrm{w} * .0}$.

Proof. If ext $S_{1} \subset(\phi(A))^{-w^{*} .0}$, then, by $w^{*} .0 .-$ compactness of $S_{1}$, and by the Krein-Milman theorem we have

$$
S_{1}=\overline{\operatorname{conv}}^{\mathrm{w}^{*} .0 .} \operatorname{ext} S_{1}=\overline{\operatorname{conv}}^{\mathrm{w}^{*} \cdot 0 .} \phi(A)=\overline{\phi(\operatorname{conv} A}^{\mathrm{w} * .0 .},
$$

and since $\phi$ is a homeomorphism in the w.o. and $w^{*}$.o. topologies on $S_{0}$ and $S_{1}$

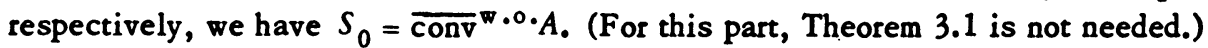

Conversely, if $S_{0}=\overline{\text { conv }}^{w} \cdot 0 \cdot A$, then, by Theorem $3.1, S_{1}={\overline{\phi(S)^{w}}}^{*} \cdot 0 \cdot=$ $\overline{\operatorname{conv}}^{*} * . \circ \cdot \phi(A)$, and, by Milman's theorem, ext $S_{1} \subset(\phi(A))^{-w^{*} .0 .}$ Q.E.D.

In $\$ 5$ we shall need an equivalent form of the above theorems. Denote by $\psi: \mathcal{L}(E, F) \rightarrow \mathfrak{L}\left(E, F^{* *}\right)$ the mapping, such that $\psi(T)=J_{F} T, T \in \mathcal{L}(E, F)$. Then, by using the natural isometry between $\mathcal{L}\left(E, F^{* *}\right)$ and $\mathcal{L}\left(F^{*}, E^{*}\right)$, the fact that this isometry composed with $\psi$ gives $\phi$, and the fact that under this isometry those spaces are $w^{*}$.o.-homeomorphic, we obtain immediately:

Corollary 3.3. Let $E, F$ be Banach spaces such that $E$ or $F^{*}$ bas the M.A.P. Then (a) $\psi(S(£(E, F)))$ is $w^{*}$.o. dense in $S\left(£\left(E, F^{* *}\right)\right)$. (b) If $A$ is a subset of $S(\&(E, F))$, then $S(\varrho(E, F))$ is the w.o.-closed convex bull of $A$ if and only if ext $S\left(£\left(E, F^{* *}\right)\right) \subset(\psi(A))^{-w^{*} . \circ .}$.

Remark. It is possible to derive a theorem of Milman type for $S(£(E, F))$, without using the dual spaces, as follows. Denote $S(\mathscr{L}(E, F))$ by $S_{0}$, and assume

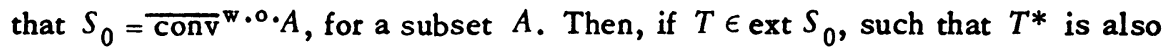
extreme, then $T \in \bar{A}^{\mathrm{w} \cdot 0 .}$. (This is seen even without Theorem 3.1, for $T^{*}$ is then

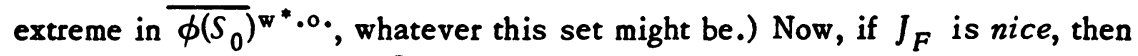
every nice operator $T$ in $\mathcal{L}(E, F)$ satisfies the above condition, for then $J_{F} T$ is also a nice operator, hence extreme, and therefore $T^{*}$ is extreme too. Hence we obtain

Proposition 3.4. If $F$ bas a nice canonical imbedding and $S(\mathscr{L}(E, F))$ is the w.o. closed convex bull of a subset $A$, then the nice operators in $\mathcal{L}(E, F)$ are in the w.o.c closure of $A$. 
Remark. Note that the canonical imbedding is always an extreme operator in $\mathcal{L}\left(E, F^{* *}\right)$, since it corresponds in the natural isometry to the identity in $\mathcal{L}\left(F^{*}, F^{*}\right)$. Examples of Banach spaces with nice canonical imbedding are the $C$-spaces and the $L^{1}$-spaces, the first by known results, and the second by the above remark and Theorem 2.2. Also, trivially, the reflexive spaces are such spaces. It is easy to produce a predual $F$ of an $L^{1}$-space, such that $J_{F}$ is not nice, but still

$$
J_{F}^{*}\left(\operatorname{ext} S\left(F^{* * *}\right)\right) \subset\left(\operatorname{ext} S\left(F^{*}\right)\right)^{-w^{*}} \text {. }
$$

We are indebted to Dr. A. J. Lazar for the following observation:

Proposition 3.5. Every Banach space F satisfies the above equation.

Proof. Let $Z=\left(\text { ext } S\left(F^{*}\right)\right)^{-w^{*}}$, equipped with the $w^{*}$-topology. We can imbed $F$ naturally into $C(Z)$, and thus $F^{* *}$ is imbedded in $C\left(Z_{0}\right)=C(Z)^{* *}$, and $F^{* * *}$ is isometric to a quotient-space of $C\left(Z_{0}\right) *$. Take $p \in$ ext $S\left(F^{* * *}\right)$. There is $q \in$ ext $S\left(C\left(Z_{0}\right)^{*}\right)$ such that the equivalence-class of $q$ is identified with $p$. With no loss of generality, we may assume $q=\delta_{x}$, for some $x \in Z_{0}$. Then $J_{F}^{*}(p)=$ $\left.J_{C(Z)}^{*}(q)\right|_{F}$. But $J_{C(Z)}^{*}(q)=\delta_{y}$, for some $y \in Z$, and $\left.\delta_{y}\right|_{F}=y$, and the result follows. Q.E.D.

4. Application to operators between $L^{1}$-spaces. In this section we apply Theorem 3.2 to operators between $L^{1}$-spaces. Let $E$ and $F$ be $L^{1}$-spaces and let $E^{*}=C\left(Y_{0}\right), F^{*}=C\left(X_{0}\right)$, for appropriate hyperstonian spaces $X_{0}, Y_{0}$. First we give a characterization of mappings of $Y_{0}$ into $X_{0}$ induced by extreme operators in $L(E, F)$ :

Lemma 4.1. The following statements are equivalent for a continuous mapping $\phi: Y_{0} \rightarrow X_{0}$ :

(a) There is a (nice) $T \in \mathscr{L}(E, F)$ such that $T^{*} f=f \circ \phi, f \in C\left(X_{0}\right)$.

(b) $\phi$ is open.

(c) For each open and dense $A \subset X_{0}, \phi^{-1}(A)$ is also dense.

Proof. (a) $\Rightarrow(c)$. One may regard $E$ (resp. $F$ ) as the space of normal measures on $Y_{0}$ (resp. $X_{0}$ ). Thus $T$ has the property that, whenever $\mu$ is normal on $Y_{0}, T \mu=\mu \circ \phi^{-1}$ is normal on $X_{0}$. But a measure on $X_{0}$ is normal if and only if it vanishes on meagre sets (cf. [2]). Hence, if $B \subset X_{0}$ is a nowhere-dense closed subset, then $\phi^{-1}(B)$ is closed and, for each $\mu \in E, \mu\left(\phi^{-1}(B)\right)=T \mu(B)=$ 0 . Thus $\phi^{-1}(B)$ is nowhere-dense, for the union of supports of normal measures on $Y_{0}$ is dense in $Y_{0}$ by [2]. Hence (c) follows.

(c) $\Rightarrow$ (a). The operator $T: C\left(Y_{0}\right) * \rightarrow C\left(X_{0}\right) *$ defined by $T \mu=\mu \circ \phi^{-1}$, $\mu \in C\left(Y_{0}\right)^{*}$, carries $E$ into $F$, for, whenever $\mu$ is normal on $Y_{0}, \mu \circ \phi^{-1}(B)=$ $\mu\left(\phi^{-1}(B)\right)=0$, for each meagre $B$, and therefore $\mu \circ \phi^{-1}$ is normal. $\left.T\right|_{E}$ is the required operator. 
(b) $\Rightarrow$ (c). This is easy and the proof is omitted.

(c) $\Rightarrow$ (b). It is sufficient to show that whenever $H \subset Y_{0}$ is clopen, then $\phi(H)$ is open. Now, $\phi(H)$ is closed and so is int $\phi(H)$, and therefore $G=$ $H \cap \phi^{-1}$ (int $\left.\phi(H)\right)$ is clopen. Now, $H \backslash G \subset \phi^{-1}[\phi(H) \backslash$ int $\phi(H)]$, and by (c) the latter is nowhere-dense. Hence $G=H$, which means that $\phi(H)=$ int $\phi(H)$ and is therefore open. Q.E.D.

Next, we prove an "intermediate" form of the theorem of Krein-Milman type for $L^{1}$-spaces, the final results to be stated in the sequel.

Proposition 4.2. With the same notations as above, the following statements are equivalent:

(a) $S(£(E, F))=\overline{\text { Conv }}^{\mathrm{w} \cdot 0 \cdot} \operatorname{ext} S(£(E, F))$.

(b) For eacb continuous mapping $\psi: Y_{0} \rightarrow X_{0}$, there is a net of open continuous mappings, $\phi_{a}: Y_{0} \rightarrow X_{0}$, such that, for every $f \in C\left(X_{0}\right), f \circ \phi_{a} \stackrel{\omega^{*}}{\longrightarrow} f \circ \psi$.

(c) Same as (b), with the condition: For each $\mu \in E$, and eacb clopen subset $A \subset X_{0}, \mu\left(\phi_{\alpha}^{-1}(A)\right) \rightarrow \mu\left(\psi^{-1}(A)\right)$.

Proof. (a) $\Rightarrow$ (b). By Theorem 3.2, we have that every extreme operator in $S\left(\mathscr{L}\left(C\left(X_{0}\right), C\left(Y_{0}\right)\right)\right)$ is in the $w^{*}$. .o.-closure of adjoints of extreme (and, by Theorem 2.2 , nice) operators in $\mathcal{L}(E, F)$. Hence, for each continuous $\psi: Y_{0} \rightarrow X_{0}$, which defines a nice positive operator $T \in \mathcal{L}\left(C\left(X_{0}\right), C\left(Y_{0}\right)\right)$, there is a net $\left\{T_{a}\right\}$ of nice operators in $\mathcal{L}(E, F)$, not necessarily positive, such that $T_{a}^{*} \stackrel{w^{*} .0 .}{\longrightarrow} T$. Now, by Lemma 4.1, for each $a$, there is $\lambda_{a} \in C\left(Y_{0}\right),\left|\lambda_{a}\right| \equiv 1$, and an open continuous mapping $\phi_{a}: Y_{0} \rightarrow X_{0}$, such that $T_{a}^{*} f=\lambda_{a} \cdot f^{\circ} \phi_{a}, f \in C\left(X_{0}\right)$. Hence, for each $f \in C\left(X_{0}\right), \lambda_{a} \cdot f \circ \phi_{a} \stackrel{\omega^{*}}{\longrightarrow} f \circ \psi$. In order to get rid of the $\lambda_{a}$ 's, assume $f$ to be real, then it is easily seen that $\left(\operatorname{Re} \lambda_{a}\right) \cdot f \circ \phi_{a} \stackrel{w^{*}}{\longrightarrow} f \circ \psi$, and in particular, $\operatorname{Re} \lambda_{a} \stackrel{w^{*}}{\longrightarrow} 1$. Let $f \in S\left(C\left(X_{0}\right)\right)$ be real. We may assume (by taking subnets if necessary) that $f \circ \phi_{a} \stackrel{w^{*}}{\longrightarrow} g$, for some $g \in C\left(Y_{0}\right)$. Now, $1-\operatorname{Re} \lambda_{a} \geq 0$ and $1 \leq$ $f \circ \phi_{a} \leq 1$ for each $a$. Hence $-\left(1-\operatorname{Re} \lambda_{a}\right) \leq\left(1-\operatorname{Re} \lambda_{a}\right) \cdot f \circ \phi_{a} \leq 1-\operatorname{Re} \lambda_{a}$, and therefore $\left(1-\operatorname{Re} \lambda_{a}\right) \cdot f \circ \phi_{a} \stackrel{w^{*}}{\longrightarrow} 0$, and it follows that $\left(\operatorname{Re} \lambda_{a}\right) \cdot f \circ \phi_{a} \stackrel{w^{*}}{\longrightarrow} g$. Thus $f \circ \psi=g$ and it follows that $f \circ \phi_{a} \stackrel{w^{*}}{\longrightarrow} f \circ \psi$, for each $f \in C\left(X_{0}\right)$.

(b) $\Rightarrow$ (a). By Theorem 3.2 and Theorem 2.2 we have to show that every extreme operator $T$ in $\mathscr{L}\left(C\left(X_{0}\right), C\left(Y_{0}\right)\right)$ is in the $\mathrm{w}^{*}$. 0.-closure of the $\mathrm{w}^{*}$-continuous nice operators. By [14, Theorem 4], every such $T$ is nice and is therefore determined by a continuous mapping $\psi: Y_{0} \rightarrow X_{0}$, if it is positive, and so (b) implies this condition for positive $T$. For a general $T$, given by $T f=\lambda \cdot f \circ \psi, f \in C\left(X_{0}\right)$, then, if $\left\{\phi_{a}\right\}$ is the net of open mappings corresponding to $\psi$, then $T_{a} f=\lambda \cdot f \circ \phi_{a}$ $f \in C\left(X_{0}\right)$, is a net of $\mathrm{w}^{*}$-continuous nice operators such that $T_{a} \stackrel{\mathrm{w}^{*} \cdot \mathrm{o}}{\longrightarrow} T$.

(b) $\Rightarrow$ (c). Take $f=X_{A}$.

(c) $\Rightarrow$ (b). Follows from the fact that the characteristic functions of clopen 
subsets in $X_{0}$ span (in norm) $C\left(X_{0}\right)$. Q.E.D.

Lemma 4.3. With the same notations as above, if, for every clopen $V \subset X_{0}$ there is an open continuous mapping of $Y_{0}$ into $V$, then (a)-(c) of Proposition 4.2 follow.

Proof. Let $\psi: Y_{0} \rightarrow X_{0}$ be a continuous mapping. Let $\sigma=\left\{A_{1}, \ldots, A_{n}\right\}$ be a partition of $X_{0}$ into a finite number of disjoint clopen subsets. For each $1 \leq$ $j \leq n$, let $\phi_{j}: Y_{0} \rightarrow A_{j}$ be an open mapping. Define $\phi^{\sigma}: Y_{0} \rightarrow X_{0}$ by

$$
\left.\phi^{\sigma}\right|_{\psi^{-1}\left(A_{j}\right)}=\left.\phi_{j}\right|_{\psi^{-1}\left(A_{j}\right)}, \quad 1 \leq j \leq n .
$$

$\phi^{\sigma}$ is clearly an open continuous mapping. Order the net $\left\{\phi^{\sigma} ; \sigma\right.$ is such a partition\}, by $\sigma_{1}>\sigma_{2}$ if $\sigma_{1}$ is a refinement of $\sigma_{2}$. If $A \subset X_{0}$ is clopen, then, for each $\sigma>\left\{A, A^{\prime}\right\}$,

$$
\left(\phi^{\sigma}\right)^{-1}(A)=\bigcup_{A_{j} \subset A, A_{j} \in \sigma}\left(\phi^{\sigma}\right)^{-1}\left(A_{j}\right)=\bigcup_{A_{j} \subset A, A_{j} \in \sigma} \psi^{-1}\left(A_{j}\right)=\psi^{-1}(A)
$$

and, obviously, (c) of Proposition 4.2 is satisfied. Q.E.D.

Theorem 4.4. For any $L^{1}$ space $E$, and any set $\Gamma$,

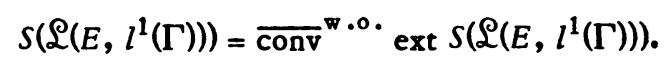

Proof. In this case $X_{0}=\beta \Gamma$, with $\Gamma$ discrete. For each clopen $V \subset X_{0}$, define $\phi: Y_{0} \rightarrow V$ such that $\phi\left(Y_{0}\right)=$ an isolated point in $V . \phi$ is clearly open, and by Lemma 4.3 the proof is completed. Q.E.D.

Theorem 4.5. If $E, F$ are $L^{1}$-spaces and ext $S(E) \neq \varnothing$, then $S(\mathscr{L}(E, F))=$ $\overline{\operatorname{conv}}^{\mathrm{w} \cdot 0 .}$ ext $S\left(\mathcal{L}(E, F)\right.$ ) if and only if $F=l^{1}(\Gamma)$, for some set $\Gamma$.

Proof. Suppose that $S(\mathscr{L}(E, F))$ is the w.o.-closed convex hull of its extreme points, and that $F$ is not of this form. Denote by $G$ the closure of the isolated points in $X_{0}$, and by $H$ the same in $Y_{0}$. By the remarks in the proof of Lemma 2.5, $H \neq \varnothing$, and by our assumption $G \neq X_{0}$. Both $G$ and $H$ are clopen. Take $x \in X_{0} \backslash G, \psi: Y_{0} \rightarrow X_{0}$ such that $\psi\left(Y_{0}\right)=\{x\}$. By Proposition 4.2, there is a net of open continuous mappings $\phi_{a}: Y_{0} \rightarrow X_{0}$ such that, for each clopen $A \subset X_{0}, \mu \in E, \mu\left(\phi_{a}^{-1}(A)\right) \rightarrow \mu\left(\psi^{-1}(A)\right)$. In particular, $\mu\left(\phi_{a}^{-1}\left(X_{0} \backslash G\right)\right) \rightarrow \mu\left(Y_{0}\right)$. But, for each $a, \phi_{a}^{-1}\left(X_{0} \backslash G\right) \cap H=\varnothing$, for otherwise it would contain an isolated $y \in H$, and then $\phi_{a}(y)$ is isolated in $X_{0} \backslash G$, which is absurd. Take an isolated $z \in Y_{0}$, then $\delta_{z} \in E$, but $0=\delta_{z}\left(\phi_{a}^{-1}\left(X_{0} \backslash G\right)\right) \nrightarrow \delta_{z}\left(Y_{0}\right)=1$. This is a contradiction which completes the proof. Q.E.D.

Example 4.6. $S \equiv S\left(\mathcal{Q}\left(l^{1}, l^{1}\right)\right)$ is not the norm-closed convex hull of its extreme points. 
Proof. Let $T \in$ ext $S$. By Lemma 2.8 there is a mapping $\phi: N \rightarrow N, \sigma \in l^{\infty}$, with $|\sigma| \equiv 1$, such that $T e_{m}=\sigma(m) e_{\phi(m)}, m \geq 1$. ( $e_{m}$ is the $m$ th unit vector in $l^{1}$.)

Define an operator $T: l^{1} \rightarrow l^{1}$ by

$$
T e_{m}=\frac{1}{m} \sum_{n=1}^{m} e_{n}, \quad m \geq 1 .
$$

$T \in S$ clearly. We shall show that the open ball of radius 1 and centre $T$ does not intersect conv ext $S$. Indeed, let $R \in$ conv ext $S$, namely, $R=\sum_{n=1}^{M} \lambda_{n} T_{n}, T_{n} \in$ ext $S, \lambda_{n} \geq 0$ for $1 \leq n \leq M$, and $\sum_{n=1}^{M} \lambda_{n}=1$. For each $n$ there is a mapping $\phi_{n}$ : $N \rightarrow N$, and $\sigma_{n} \in l^{\infty}$, with $\left|\sigma_{n}\right| \equiv 1$, such that, for each $m, R e_{m}=\sum_{n=1}^{M} \lambda_{n} \sigma_{n}(m) e_{\phi_{n}(m)}$ and therefore

$$
\left(R e_{m}\right)_{j}=\sum_{\phi_{n}(m)=j} \lambda_{n} \sigma_{n}(m), \quad j \geq 1 .
$$

Now, $\|R-T\|=\sup _{m \geq 1}\left\|R e_{m}-T e_{m}\right\|$. But, for each $m$,

Define

$$
\left\|R e_{m}-T e_{m}\right\|=\sum_{j=1}^{m}\left|\frac{1}{m}-\sum_{\phi_{n}(m)=j} \lambda_{n} \sigma_{n}(m)\right|+\sum_{j>m}\left|\sum_{\phi_{n}(m)=j} \lambda_{n} \sigma_{n}(m)\right| .
$$

$$
\delta=\min \left\{\sum_{n=1}^{M} a_{n} \lambda_{n} ; \sum_{n=1}^{M} a_{n} \lambda_{n}>0, a_{n}=+1,0,-1,1 \leq n \leq M\right\}>0 .
$$

Take $m$ such that $1 / m<\delta / 2$, and then

$$
\left\|R e_{m}-T e_{m}\right\| \geq \sum_{j=1}^{m}\left|\frac{1}{m}-\sum_{\phi_{n}(m)=j} \lambda_{n} \sigma_{n}(m)\right| \geq \sum_{j=1}^{m} \frac{1}{m}=1 .
$$

Thus $\|R-T\| \geq 1$. Q.E.D.

Now we pass to the case where ext $S(E)=\varnothing$. This means that if we represent $E$ as an $L^{1}(\mu)$-space, then $\mu$ is purely nonatomic. If we combine the decompositions of Kakutani [9] and Maharam [11], it follows that $E$ is isometric to a direct sum $\left(\Sigma_{a} L^{1}[0,1]^{n_{a}}\right)_{1}$ for a suitable family $\left\{n_{a}\right\}$ of infinite cardinals. (By $L^{1}[0,1]^{m}$ we mean the space of integrable functions on $[0,1]^{m}$ with the Lebesgue product measure, cf. [11] or [13].) Thus, $E^{*}=C\left(Y_{0}\right)$ is isometric to $\left(\Sigma_{a} L^{\infty}[0,1]^{n_{\alpha}}\right)_{\infty}$. For any infinite cardinal $m$, denote by $Z_{m}$ the hyperstonian space satisfying $C\left(Z_{m}\right)=L^{\infty}[0,1]^{m}$. For each $\alpha$, there is a natural projection $P_{a}: C\left(Y_{0}\right) \rightarrow$ $C\left(Z_{n_{a}}\right)$, which is multiplicative, $w^{*}$-continuous, and $P_{a} 1=1$. Hence, there is an open homeomorphism (cf. Lemma 4.1) of $Z_{n_{a}}$ into $Y_{0}$, and so $Z_{n_{a}}$ may be regarded as a clopen subset of $Y_{0}$. It is easily checked that $Z_{n_{a}} \cap Z_{n_{\beta}}=\varnothing$ whenever $\alpha \neq \beta$, and that $U_{a} Z_{n_{a}}$ is dense in $Y_{0}$. Another important property of the spaces $Z_{m}$ is their bomogeneity, i.e., every clopen subset of $Z_{m}$ is homeomorphic to $Z_{m^{*}}$ This is an easy interpretation of Maharam's results [11], for whenever there is an isomorphism $r:\left(\Sigma_{1}, \mu_{1}\right) \rightarrow\left(\Sigma_{2}, \mu_{2}\right)$ between two measure algebras, it induces an 
isometry $T$ of $L^{\infty}\left(\Sigma_{1}, \mu_{1}\right)$ onto $L\left(\Sigma_{2}, \mu_{2}\right)$ such that $T \chi_{A}=\chi_{T(A)}$, for each $A \in \Sigma_{1}$, and so the maximal-ideal spaces of the $L^{\infty}$-spaces are homeomorphic.

The following important lemma is the main tool in our investigation:

Lemma 4.7. Let $m, n$ be two infinite cardinals, and let $Z_{m}, Z_{n}$ be the spaces defined above. Then the following statements are equivalent:

(a) $m \geq n$.

(b) $Z_{n}$ is an open continuous image of $Z_{m}$.

(c) $Z_{n}$ is a continuous image of $Z_{m}$.

Proof. $(a) \Rightarrow(b)$. Let $I$ be a set of cardinality $m, J \subset I$ a set of cardinality n. Then $[0,1]^{m}=\Pi_{i \in I}[0,1]_{i}$ and $[0,1]^{n}=\Pi_{i \in J}[0,1]_{i}$, where $[0,1]_{i}$ denotes, for each $i$, the interval $[0,1]$. For each measurable $E$ in $[0,1]^{n}$, define a measurable $\widetilde{E}$ in $[0,1]^{m}$ such that $\widetilde{E}=E \times \Pi_{i \in I \backslash}[0,1]_{i}$. Denote the product measure on $[0,1]^{m}$ (resp. $[0,1]^{n}$ ) by $\mu_{m}$ (resp. $\mu_{n}$ ). Now, let $f \in L^{1}[0,1]^{m}$, and define a measure $\nu$ on $[0,1]^{n}$ by $\nu(E)=\int_{\widetilde{E}} / d \mu_{m}$, for each measurable $E$ in $[0,1]^{n}$. Clearly $\nu$ is absolutely continuous with respect to $\mu_{n}$, and therefore $d \nu=$ $(P f) d \mu_{n}$, where $P f \in L^{1}[0,1]^{n}$. Thus we have defined a linear operator $P$ of norm 1 from $L^{1}[0,1]^{m}$ onto $L^{1}[0,1]^{n}$. Now $P^{*} \chi_{E}=\chi_{\widetilde{E}}$, for each measurable subset $E$ of $[0,1]^{n}$, and hence $P^{*} 1=1$, and $P^{*}$ is easily seen to be multiplicative. Thus, by Lemma 4.1, there is an open continuous mapping of $Z_{m}$ onto $Z_{n}$ (the same $P$ was constructed in a somewhat different way in $[13, \mathrm{p} .220]$ ).

(b) $\Rightarrow$ (c). Trivial.

(c) $\Rightarrow$ (a). Let $\psi$ be a continuous mapping of $Z_{m}$ onto $Z_{n}$. The operator $T: C\left(Z_{n}\right) \rightarrow C\left(Z_{m}\right)$, given by $T f=f \circ \psi, f \in C\left(Z_{n}\right)$, is a bounded one-to-one operaator. Now, by [13, Corollary 3.3] and the remark following Theorem 3.5 of [13], it follows that $m \geq n$. Q.E.D.

Remark. By the remark following the proof of Theorem 3.5 of [13], it follows that the weight (i.e., the minimal cardinality of a base for the clopen subsets) of $Z_{m}$, as well as the cardinality of the set of all clopen subsets of $Z_{m}$, is equal to $m^{N_{0}}$. For weight $\left(Z_{m}\right)=\operatorname{dim} C\left(Z_{m}\right)=\operatorname{dim} L^{\infty}[0,1]^{m}=m^{K_{0}}$, and since the cardinality of $L^{\infty}[0,1]^{m}$ is also $m^{x_{0}}$, the second statement follows. By the same remark, for any infinite cardinal $p$, there are cardinals $m, n \geq p$, such that $m>n$ and $m^{X_{0}}=n^{X_{0}}$. Thus, although the spaces $Z_{m}, Z_{n}$ have the same weight, the same cardinality of the set of their clopen subsets, and they are both homogeneous yet there is no continuous mapping of $Z_{n}$ onto $Z_{m}$. It is likely that the cardinality of $Z_{m}$ is greater than that of $Z_{n}$, whenever $m>n$. If this were true, then the above remark becomes trivial, and the proof of Lemma 4.7 would be considerably simplified. However, we have not been able to prove that.

Let $E, F$ be $L^{1}$-spaces with no extreme points in their unit balls. Put 
$E^{*}=C\left(Y_{0}\right), F^{*}=C\left(X_{0}\right)$ and decompose $X_{0}, Y_{0}$ in the form $X_{0}=\overline{U_{a \in A} Z_{n_{a}}}$, $Y_{0}={\overline{U_{\beta \epsilon B} Z_{m} \beta^{*}}}$ The decompositions need not be unique, since $C\left(Z_{n}\right)$ is isometric to $\left(C\left(Z_{n}^{\beta}\right) \oplus C\left(Z_{n}\right) \oplus \ldots\right)_{\infty}$, for each infinite cardinal $n$, and therefore we may repeat each $Z_{n_{a}}$ countably many times in $X_{0}$.

Corollary 4.8. With the above notations, $Y_{0}$ is an open continuous image of $X_{0}$ if and only if there is a surjection $w: A \rightarrow B$ such that, for each $\alpha \in A$, $n_{a} \geq m_{w(\alpha)}$

Proof. If the latter condition is satisfied, we know by Lemma 4.7 that for each $a$ there is an open continuous mapping $\phi_{a}$ of $Z_{n_{a}}$ onto $Z_{m_{w}(a)}$. Define $\phi: X_{0} \rightarrow Y_{0}$ by $\phi \mid z_{n_{a}}=\phi_{a}, a \in A$. Since $X_{0}$ is the Stone-Čech compactification of $\bigcup_{a \in A} Z_{n_{a}}$, $\phi$ is well defined and is continuous and onto. To see that $\phi$ is open, let $V \subset X_{0}$ be clopen. Then $V=\bar{U}_{a \in A}\left(V \cap Z_{n_{a}}\right)$, and therefore $\phi(V)=$ $\bar{U}_{a \in A} \phi\left(V \cap Z_{n_{a}}\right)$. But $\phi\left(V \cap Z_{n_{a}}\right)$ is open for each $a$, and so $\phi(V)$ is also open. Conversely, if there is an open continuous mapping $\phi$ of $X_{0}$ onto $Y_{0}$, then fix $\alpha \in A$. We claim that $\phi\left(Z_{n_{a}}\right)$ intersects only countably-many $Z_{m}$ 's. For every such intersection is clopen and its inverse image is clopen in $Z_{n_{a}}$. The family of all such nonvoid inverse images is a family of pairwise disjoint clopen subsets of $Z_{n_{\alpha}}$. But this space satisfies the countable-chain-condition, since it carries a strictly positive measure, namely the Lebesgue product measure $\mu_{n_{a}}$, regarded as a normal measure on $Z_{n_{a}}$ (cf. [3]). Hence, that family is at most countable. But if $\phi\left(Z_{n_{a}}\right) \cap Z_{m_{\beta}} \neq \varnothing$, then, by homogeneity of those spaces, $Z_{m_{\beta}}$ is an open continuous image of $Z_{n_{a}}$, and, by Lemma 4.7, $n_{a} \geq m_{\beta}$. By assumptions, $Z_{n_{a}}$ has countably many copies in $X_{0}$, and the image of their union still intersects only countably many $Z_{m}$ 's. Thus, we define $w$ on the set of indices of those copies, by assigning to each such index one of the $\beta$ 's of the above statement, and covering this way all such $\beta$ 's. In this way we obtain the required surjection $w$. Q.E.D.

Corollary 4.9. (a) The Yosida-Hewitt space, i.e. the maximal-ideal space of $L^{\infty}[0,1]$, is the only byperstonian perfect space which is an open continuous image of every byperstonian perfect space.

(b) Suppose that $Z$ is a byperstonian perfect space, with the property that for every clopen $V \subset Z$, there is an open continuous mapping of $Z$ into $V$. If $Z$ satisfies the countable-chain-condition, then $Z$ is homogeneous. Otberwise, there is a bomogeneous space W such that a union of disjoint clopen copies of $W$ is dense in $Z$.

Proof. (a) By [11], the Yosida-Hewitt space is simply $Z_{\boldsymbol{X}_{0}}$, and the result is immediate from Corollary 4.8 . 
(b) The second assertion follows immediately from Corollary 4.8. The first one is implied by the fact that the number of such copies is at most countable. Q.E.D.

Now we are in position to prove the theorem of Krein-Milman type in the nonatomic case. Let $E$ and $F$ be $L^{1}$-spaces, with ext $S(E)=\varnothing$. Decompose $E$ into the sum $\left(\Sigma_{a \in A} L^{1}[0,1]^{n_{a}}\right)_{1}$, and $F$ into the sum $\left(l^{1}(\Gamma) \oplus \Sigma_{\beta \in B} L^{1}[0,1]^{m}\right)_{1}$, for suitable families $\left\{n_{a}\right\}_{a \in A},\left\{m_{\beta}\right\}_{\beta \in B}$ of infinite cardinals, and a set $\Gamma$.

Theorem 4.10. A necessary and sufficient condition for $S(\varrho(E, F))(E, F$ as above) to be the w.o.-closed convex bull of its extreme points is that $n_{\alpha} \geq m_{\beta}$ for each $a \in A, \beta \in B$.

Proof. Put $E^{*}=C\left(Y_{0}\right), F^{*}=C\left(X_{0}\right)$. The above decompositions imply that $Y_{0}=\overline{U_{n_{\alpha}}}$ and $X_{0}=\overline{Z_{m_{\beta}}} \cup H$, where $H$ is the closure of the isolated points in $X_{0}$. If the condition is satisfied, then we make use of Lemma 4.3. Take a clopen $V \subset X_{0}$. If $V \cap H \neq \varnothing$, then obviously there is an open continuous mapping of $Y_{0}$ into $V$. Otherwise, there is $\beta \in B$, such that $V \cap Z_{m_{\beta}} \neq \varnothing$, and, by homogeneity, it is sufficient to show that $Z_{m_{\beta}}$ is an open continuous image of $Y_{0}$, and this follows from Corollary 4.8 .

Conversely, if $S(\mathscr{L}(E, F))=\overline{\text { conv }}^{\text {w.0. ext }} S(\mathscr{Q}(E, F))$, then, by Proposition 4.2, for each $z \in X_{0} \backslash H$, there is a net of open continuous mappings $\phi_{\gamma}: Y_{0} \rightarrow$ $X_{0}$, such that, for every clopen $V \subset X_{0}, \chi_{\phi} \bar{\gamma}^{-1}(V) \stackrel{w^{*}}{\rightarrow} \chi_{\phi^{-1}(V)}$, where $\phi: Y_{0} \rightarrow$ $X_{0}$ is a constant mapping such that $\phi\left(Y_{0}\right)=\{z\}$. In particular, fix $\beta \in B$, and take $V=Z_{m_{\beta}}, z \in V$. Then $\chi_{\phi_{\gamma}^{-1}\left(Z_{m \beta}\right)} \stackrel{w^{*}}{\longrightarrow} 1$. Hence, for each $a \in A, \phi_{\gamma}^{-1}\left(Z_{m_{\beta}}\right) \cap$ $Z_{n_{a}} \neq \varnothing$, for some $\gamma$, for otherwise $\chi_{\phi}{ }_{\gamma}^{-1}\left(Z_{m \beta}\right)$ should not converge-w* to 1 . Hence, by homogeneity, $Z_{m}$ is an open continuous image of $Z_{n_{a}}$, and by Lemma 4.7, $n_{a} \geq m_{\beta}$ for each $\alpha \in A, \beta \in B$. Q.E.D.

Remark. The results of Theorems 4.4, 4.5, and 4.10, can be unified, if we define $Z_{0}$ to be a one-point space, and when decomposing a hyperstonian space $Y_{0}$ into $\overline{U_{a} Z_{n_{a}}}$, we allow $n_{a}$ to be an infinite cardinal, or 0 . Then the formulation of Theorem 4.10 applies to the general case as well.

Corollary 4.11. Let $E$ be an $L^{1}$ space. Then $S(\mathscr{L}(E, E))$ is the w.o..closed convex bull of its extreme points if and only if either $E=l^{1}(\Gamma)$, for some set $\Gamma$, or $E^{*}$ bas a maximal ideal space as in Corollary 4.9(b).

Proof. Immediate.

5. Various applications. In this section we give some other applications of the results of $\$ 3$.

Our first result deals with extreme operators in $\mathscr{L}\left(l^{1}(\Gamma), E\right)$, where $E$ is an 
arbitrary Banach space. For the characterization of those operators see Lemma 2.8. We also need the following trivial lemma:

Lemma 5.1. If $E$ is a Banach space, then $S(E)=\overline{\operatorname{conv}}$ ext $S(E)$ if and only

$$
\operatorname{ext} S\left(E^{* *}\right) \subset\left(J_{E}(\operatorname{ext} S(E))\right)^{-w^{*}} .
$$

Proof. Though it is easy to prove this in elementary ways, it is interesting to apply Theorem 3.2. For if $K$ is a one-dimensional space, then $S(E)=$ conv ext $S(E)$ if and only if $S(\mathscr{Q}(K, E))$ is the w.o.-closed convex hull of its extreme points, and this is equivalent to ext $S\left(E^{* *}\right)=\operatorname{ext} S\left(\mathscr{L}^{*}\left(E^{*}, K^{*}\right)\right) \subset$ $\left(J_{E}(\text { ext } S(E))\right)^{-w^{*}}$, for $J_{E}$ is precisely the injection of $E=\mathscr{L}(K, E)$ into $\mathscr{L}\left(E^{*}, K^{*}\right)$ $=E^{* *}$, and the $\mathrm{w}^{*}$.o.-topology in $\mathscr{L}\left(E^{*}, K^{*}\right)$ is precisely the $\mathrm{w}^{*}$-topology in $E^{* *}$. Q.E.D.

Theorem 5.2. Let $E$ be a Banach space. Then $S\left(£\left(l^{1}(\Gamma), E\right)\right)$ is the w.o.closed convex bull of its extreme points if and only if $S(E)$ is the norm-closed convex bull of its extreme points.

Proof. If $S(E)=\overline{\text { conv }}$ ext $S(E)$, then, by Lemma 5.1, ext $S\left(E^{* *}\right) C$ $\left(J_{E}(\text { ext } S(E))\right)^{-w^{*}}$. Let $R: E^{*} \rightarrow l^{\infty}(\dot{\Gamma})$ be an extreme operator. By Theorem 3.2 we have to show the existence of a net of extreme operators $T_{a}: l^{1}(\Gamma) \rightarrow E$, such that $T_{a}^{*} \stackrel{\mathbb{W}^{*} \cdot 0}{\longrightarrow} R$. Since $\left\{e_{\gamma}\right\}_{\gamma \in \Gamma}$ span $l^{1}(\Gamma)$ in norm, it is sufficient to check that, for each $\gamma \in \Gamma, x^{*} \in E^{*}, x^{*}\left(T_{a^{e}} \gamma_{\gamma}\right) \rightarrow\left\langle R x^{*}, e_{\gamma}\right\rangle=R^{*} \gamma^{*}\left(x^{*}\right)$. (Note that $R$ is defined by a w*-continuous mapping $R^{*}: \beta \Gamma \rightarrow E^{* *}$.) That is: $\left.J_{E}\left(T_{a} e\right)\right) \stackrel{\mathrm{w}^{*}}{\longrightarrow}$ $R^{*} \gamma$ for each $\gamma \in \Gamma$. But for each $\gamma \in \Gamma$ there is a net $\left\{x_{\beta_{y}}\right\}_{\beta_{y} \in A y} C$ ext $S(E)$, such that $J_{E^{x}} \beta_{y} \stackrel{\stackrel{*}{*}^{*}}{\longrightarrow} R^{*} \gamma$ (because $R^{*} \gamma$ is in ext $S\left(E^{* *}\right)$ for each $\gamma \in \Gamma$, since $R$ is extreme). Take $A=\Pi_{\gamma} A_{y}$ with the natural ordering, and for each $a=$

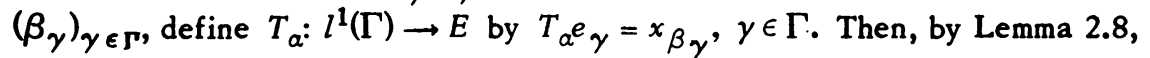
$T_{a}$ is extreme in $\mathscr{L}\left(l^{1}(\Gamma), E\right)$ and we have $J_{E}\left(T_{a} e_{\gamma}\right) \stackrel{w^{*}}{\longrightarrow} R^{*} \gamma$, for each $\gamma \in \Gamma$.

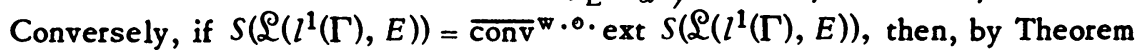
3.2, for each extreme $R: E^{*} \rightarrow l^{\infty}(\Gamma)$, there is a net of extreme operators $T_{a}$ : $l^{1}(\Gamma) \rightarrow E$, such that $J_{E}\left(T_{\alpha^{e}}{ }\right) \stackrel{w^{*}}{\longrightarrow} R^{*} \gamma$, for each $\gamma \in \Gamma$. In particular, take $z \epsilon \operatorname{ext} S\left(E^{* *}\right)$, and let $R$ be the extreme operator induced by the mapping $R^{*}(\Gamma)=$ $\{z\}$. Since, by Lemma 2.8, $T_{a} e \gamma \in$ ext $S(E)$ for each $\alpha, \gamma$, we obtain

$$
\operatorname{ext} S\left(E^{* *}\right) \subset\left(J_{E}(\operatorname{ext} S(E))\right)^{-w^{*}},
$$

and the proof is completed by Lemma 5.1. Q.E.D.

Remark. The "only if" part of Theorem 5.2 bears the following generaliza-

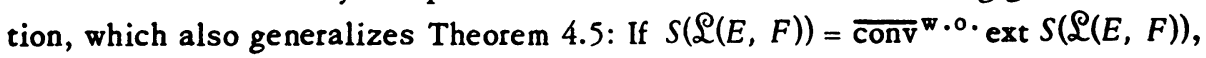
where $E$ is an $L^{1}$-space with ext $S(E) \neq \varnothing$, and $F$ an arbitrary Banach space, then $S(F)=\overline{\text { conv }}$ ext $S(F)$. 
Proof. Put $E^{*}=C\left(Y_{0}\right)$, and define $R: F^{*} \rightarrow C\left(Y_{0}\right)$ by the mapping $R^{*}\left(Y_{0}\right)=$ $\{z\}$, for some $z \in \operatorname{ext} S\left(F^{* *}\right)$. Then $R$ is extreme, and by Theorem 3.2, there is a net of extreme operators $T_{a}: E \rightarrow F$, such that $T_{a}^{*} \stackrel{w^{*} .0}{\longrightarrow} R$. In particular, for each $p \in \operatorname{ext} S(E)$, if we write $J_{E} p=\delta_{y}$, for some isolated $y \in Y_{0}$, then $J_{F}\left(T_{a} p\right) \stackrel{w^{*}}{\longrightarrow}$ $R^{*} y=Z$. By Theorem 2.9, $T_{\alpha} p \in$ ext $S(F)$, for each $\alpha$, and since ext $S(E)$ is nonvoid, the proof is completed. Q.E.D.

Our next result deals with operators into $C$-spaces. By applying Corollary 3.3 to these operators, we obtain a necessary and sufficient condition for $S(\mathcal{L}(E, C(Y)))$ to be the w.o.-closed convex hull of its nice operators. This is an improvement of the results of Morris and Phelps [12]. We tried as much as possible to set this condition in terms of the topological properties of $Y$ and ext $S\left(E^{*}\right)$, as did Morris and Phelps, but the reader may find other such equivalent conditions, by the proof of Theorem 5.4.

Definition. Let $Y$ be a compact Hausdorff space, $E$ a Banach space, and $Z$ a subset of $E^{*}$. We say that the pair $(Y, Z)$ has property $\left(D_{1}\right)$ if, given $\epsilon>0$, $x_{1}, \cdots, x_{n} \in E$,

$$
\mu_{1}, \ldots, \mu_{n} \in C(Y)^{*}, p_{1}, \cdots, p_{m} \in Z \text { and } K_{1}, \ldots, K_{m} \subset Y
$$

pairwise-disjoint and compact subsets, then there exists a $w^{*}$-continuous mapping $\phi: Y \rightarrow Z$ such that, for each $i, j$,

$$
\left|\int_{K_{j}}\left\langle\phi(y)-P_{j}, x_{i}\right\rangle d \mu_{i}(y)\right|<\epsilon\left|\mu_{i}\right|\left(K_{j}\right) .
$$

It is easily checked that property (D) of [12] implies property $\left(D_{1}\right)$, and that property $\left(D_{1}\right)$ implies the pointwise density of the continuous mappings of $Y$ into $Z$ in $(Z)^{Y}$.

Now, let $Y$ be a compact Hausdorff space, and put, as usual, $C(Y)^{* *}=$ $C\left(Y_{0}\right), J_{C(Y)}=J$. We have the following technical lemma.

Lemma 5.3. For each clopen $A \subset Y_{0}, \chi_{A}$ is in the $w^{*}$-closure of $\left\{\chi_{\text {int } J^{*}-1(K)}: K \subset Y\right.$ is compact $\}$.

Proof. It is sufficient to show that $S\left(C\left(Y_{0}\right)\right)=\overline{\operatorname{conv}}^{{ }^{*}}\left\{2 \chi_{\text {int } J^{*-1}(K)}-1\right.$ : $K \subset Y$ is compact $\}$, for then the result follows by Milman's theorem. If $\mu \in C(Y)^{*}$, denote by $\hat{\mu}$ the corresponding normal measure on $Y_{0}$. Observe that if $K \subset Y$ is compact, then $\mu(K)=\hat{\mu}\left(\right.$ int $J^{*-1}(K)$ ). (This is seen, e.g., by taking a decreasing net $\left\{f_{a}\right\} \subset C(Y)$, such that $\left.f_{a}\right|_{K} \equiv 1$, and $f_{a} \backslash \chi_{K}$ pointwise. The net $\left\{J f_{a}\right\}$ is decreasing in $C\left(Y_{0}\right)$ and so converges- $w^{*}$, and its limit is easily seen to be $\chi_{\text {int } J^{*-1}(K)}$. The above assertion follows from the fact that $\mu$ is regular and $\hat{\mu}$ is normal.) Now, for each $\epsilon>0, f \in S(C(Y)), \mu_{1}, \cdots, \mu_{n} \in C(Y)^{*}$, there are 
pairwise-disjoint and compact subsets $K_{1}, \ldots, K_{m}$ of $Y$, such that

$$
\left|\int_{Y}\left(f-\sum_{i=1}^{m} \lambda_{i} x_{K_{i}}\right) d \mu_{j}\right|<\epsilon, \quad 1 \leq j \leq n, \text { where }-1 \leq \lambda_{i} \leq 1 \text {. }
$$

It is easily seen that this combination may be replaced by a convex combination of the desired form. This completes the proof, for $S(C(Y))$ is $w^{*}$-dense in $S\left(C\left(Y_{0}\right)\right)$. Q.E.D.

Theorem 5.4. Let $Y$ be a compact Hausdorff space and $E$ a Banacb space. Then $S(\mathscr{L}(E, C(Y)))$ is the w.o.-closed convex bull of its nice operators if and only if the pair $\left(Y\right.$, ext $\left.S\left(E^{*}\right)\right)$ bas property $\left(D_{1}\right)$.

Proof. Assume that $\left(Y\right.$, ext $\left.S\left(E^{*}\right)\right)$ has property $\left(D_{1}\right)$. It is sufficient to show

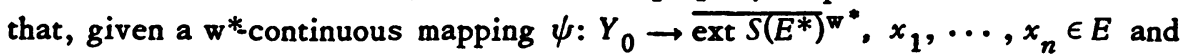
$\mu_{1}, \cdots, \mu_{n} \in C(Y)^{*}$, there is a $w^{*}$-continuous mapping $\phi: Y \rightarrow \operatorname{ext} S\left(E^{*}\right)$, such that

$$
\left|\int_{Y_{0}}\left\langle\psi(y), x_{i}\right\rangle d \hat{\mu}_{i}(y)-\int_{Y}\left\langle\phi(y), x_{i}\right\rangle d \mu_{i}(y)\right|<1, \quad 1 \leq i \leq n .
$$

(As above, $\hat{\mu}_{i}$ is $\mu_{i}$ as a normal measure on $Y_{0}$.) For, having shown this, then this condition is satisfied by every $\psi$ induced by an extreme operator in $\mathcal{L}\left(E, C\left(Y_{0}\right)\right.$ ) (by [14], since $Y_{0}$ is stonian), and this means that every such operator is in the $w^{*}$.o.-closure of $\{J T: T \in \mathcal{L}(E, C(Y))$ is nice\}, and by Corollary 3.3 the result follows. With no loss of generality we may assume that $\mu_{i}>0,1 \leq i \leq n$. For each $p \in \operatorname{ext} S\left(E^{*}\right)$, define

$$
V_{p}=\left\{y \in Y_{0}:\left|\left\langle\psi(y)-p, x_{i}\right\rangle\right|<1 / 3\left\|\mu_{i}\right\|, 1 \leq i \leq n\right\} \text {. }
$$

Then $\left\{V_{p}: p \in\right.$ ext $\left.S\left(E^{*}\right)\right\}$ is an open cover of $Y_{0}$. Hence, there is a decomposition $Y_{0}=\bigcup_{j=1}^{m} A_{j}$ into a finite number of clopen disjoint subsets, such that for each $1 \leq j \leq m$, there is $p_{j} \in$ ext $S\left(E^{*}\right)$ such that

$$
A_{j} \subset\left\{y \in Y_{0}:\left|\left\langle\psi(y)-P_{j}, x_{i}\right\rangle\right| \leq 1 / 3\left\|\mu_{i}\right\|, 1 \leq i \leq n\right\} .
$$

Hence we have

$$
\left|\int_{Y_{0}}\left\langle\psi(y)-\sum_{j=1}^{m} \chi_{A}(y) p_{j}, x_{i}\right\rangle d \hat{\mu}_{i}(y)\right| \leq \frac{1}{3}, \quad 1 \leq i \leq n .
$$

By Lemma 5.3, there are compact subsets $K_{1}, \ldots, K_{m} \subset Y$, such that

$$
\hat{\mu}_{i}\left(A_{j} \Delta \text { int } J^{*-1}\left(K_{j}\right)\right)<1 / 12 m(m+1)\left\|x_{i}\right\|, \quad 1 \leq i \leq n \text { and } 1 \leq j \leq m .
$$

(This follows from the fact that $\chi_{A \Delta B}=\chi_{A}+\chi_{B}-2 \chi_{A} \cdot \chi_{B}$ and the right side converges- $w^{*}$ to zero whenever $\chi_{B}$ converges-w* to $\chi_{A}$.) 
Since the $A_{j}$ 's are pairwise-disjoint, it is easily verified that $\mu_{i}\left(K_{j} \cap\left(U_{s \neq j} K_{s}\right)\right)<1 / 12(m+1)\left\|x_{i}\right\|$, for each $1 \leq i \leq n, 1 \leq j \leq m$. We may therefore replace each $K_{j}$ by a compact $L_{j} \subset K_{j} \backslash \bigcup_{s \neq j} K_{s}$, with $\mu_{i}\left(K_{j} \backslash L_{j}\right)<$ $1 / 12(m+1)\left\|x_{i}\right\|+1 / 12 m\left\|x_{i}\right\|, i=1, \ldots, n$. Hence, $\hat{\mu}_{i}\left(A_{j} \Delta\right.$ int $\left.J^{*-1}\left(L_{j}\right)\right)<$ $1 / 6 m\left\|x_{i}\right\|$, for $i=1, \ldots, n$ and $j=1, \ldots, m$. In particular we have

$$
\left\|\mu_{i}\right\|-\mu_{i}\left(\bigcup_{j=1}^{m} L_{j}\right)<\frac{1}{6\left\|x_{i}\right\|}, \quad i=1, \ldots, n .
$$

By property $\left(D_{1}\right)$ there is a continuous mapping $\phi: Y \rightarrow \operatorname{ext} S\left(E^{*}\right)$ satisfying

$$
\left|\int_{L_{j}}\left\langle\phi(y)-p_{j}, x_{i}\right\rangle d \mu_{i}(y)\right|<\frac{1}{3\left\|\mu_{i}\right\|} \mu_{i}\left(K_{j}\right), \quad 1 \leq i \leq n, 1 \leq j \leq m .
$$

Putting (5.2)-(5.4) together, we obtain (5.1).

Conversely, if $S(\mathscr{Q}(E, C(Y)))$ is the w.o.-closed convex hull of its nice operators, then take $\epsilon>0, x_{1}, \ldots, x_{n} \in E, \mu_{1}, \cdots, \mu_{n} \in C(Y)^{*}, p_{1}, \cdots, p_{m} \epsilon$ ext $S\left(E^{*}\right)$ and $K_{1}, \cdots, K_{m} \subset Y$ pairwise-disjoint and compact. Set $A_{j}=$ int $J^{*-1}\left(K_{j}\right)$, for $1 \leq j \leq m$, and let $A_{0}$ be $Y_{0} \backslash \bigcup_{j=1}^{m} A_{j}$, and $p_{0}$ be an arbitrary element of ext $S\left(E^{*}\right)$. Now $\psi=\sum_{j=0}^{m} \chi_{A_{j}} p_{j}$ is a $w^{*}$-continuous mapping of $Y_{0}$ into ext $S\left(E^{*}\right)$. By Corollary 3.3 there is a $w^{*}$-continuous mapping $\phi: Y \rightarrow Z$ such that

$$
\left|\int_{Y_{0}}\left\langle\psi(y), x_{i}\right\rangle d \hat{\mu}_{i j}(y)-\int_{Y}\left\langle\phi(y), x_{i}\right\rangle d \mu_{i j}(y)\right|<\epsilon\left|\mu_{i}\right|\left(K_{j}\right),
$$

for each $1 \leq i \leq n, 1 \leq j \leq m$, where $\mu_{i j}=\left.\mu_{i}\right|_{K_{j}}$. Namely

$$
\left|\int_{K_{j}}\left\langle\phi(y)-p_{j}, x_{i}\right\rangle d \mu_{i}(y)\right|<\epsilon\left|\mu_{i}\right|\left(K_{j}\right), \quad 1 \leq i \leq n, 1 \leq j \leq m . \quad \text { Q.E.D. }
$$

\section{REFERENCES}

1. R. M. Blumenthal, J. Lindenstrauss and R. R. Phelps, Extreme operators into $C(K)$, Pacific J. Math. 15 (1965), 747-756. MR 35 \#758.

2. J. Dixmier, Sur certains es paces considéres par M. H. Stone, Summa Brasil. Math. 2 (1951), 151-182. MR 14, 69.

3. —, Sur un théorème de Banach, Duke Math. J. 15 (1948), 1057-1071. MR 10, 306.

4. N. Dunford and J. T. Schwartz, Linear operators. I: General theory, Pure and Appl. Math., vol. 7, Interscience, New York, 1958. MR 22 \#8302.

5. L. Gillman and M. Jerison, Rings of continuous functions, University Series in Higher Math., Van Nostrand, Princeton, N. J., 1960. MR 22 \#6994.

6. A. Grothendieck, Produits tensoriels topologique et espaces nucleaires, Mem. Amer. Math. Soc. No. 16 (1955). MR 17, 763.

7. - Une charactérisation vectorielle métrique des espaces $L^{1}$, Canad. J. Math. 7 (1955), 552-561. MR 17, 877. 
8. W. B. Johnson, H. P. Rosenthal and M. Zippin, On bases, finite dimensional decompositions and weaker structures in Banach spaces, Israel J. Math. 9 (1971), 488-506. MR $43 \# 6702$.

9. S. Kakutani, Concrete representations of abstract $(L)$-spaces and the mean ergodic theorem, Ann. of Math. (2) 42 (1941), 523-537. MR 2, 318.

10. J. Lindenstrauss, Extension of compact operators, Mem. Amer. Math. Soc. No. 48 (1964). MR 31 \#3828.

11. D. Maharam, On homogeneous measure algebras, Proc. Nat. Acad. Sci. U. S. A. 28 (1942), 108-111. MR 4, 12.

12. P. D. Morris and R. R. Phelps, Theorems of Krein-Milman type for certain convex sets of operators, Trans. Amer. Math. Soc. 150 (1970), 183-200. MR 41 \#7409.

13. H. P. Rosenthal, On injective Banach spaces and the spaces $L^{\infty}(\mu)$ for finite

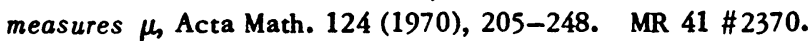

14. M. Sharir, Characterization and properties of extreme operators into $C(Y)$, Israel J. Math. 12 (1972), 174-183.

15. K. Sundaresan, Extreme points of the unit cell in Lebesgue-Bochner function spaces, Colloq. Math. 22 (1970), 111-119. MR 43 \#2493.

16. S. Sakai, $C^{*}$ algebras and $w^{*}$-algebras, Ergebnisse der Math. und ihrer Grenzgebiete, vol. 60, Springer-Verlag, Berlin and New York, 1971.

DEPARTMENT OF MATHEMATICAL SCIENCES, TEL AVIV UNIVERSITY, TEL AVIV, ISRAEL 